\title{
Metric properties of $\varepsilon$-trajectories of dynamical systems with stochastic behaviour
}

\author{
M. L. BLANK \\ All-Union Research Centre of Cardiology, AMS, 3 Cherepkovsky Street 15, Moscow \\ 121552, USSR
}

(Received 14 November 1986 and revised 1 April 1987)

\section{Introduction}

A highly developed branch of the modern theory of dynamical systems is the study of deterministic ones with statistical properties in behaviour. During the last decade such systems were discovered in various domains of physics, chemistry, biology and technology. It is due to their complexity, that only the simplest of such systems have been analytically investigated (Lorenz system, Rikitake dynamo, billiard systems), and that is why numerical methods are widely used, especially in applied investigations. In numerical modelling we have no true trajectory of a dynamical system $f$, but an approximation $\bar{x}=\left(x_{1}, x_{2}, \ldots\right)$ such that the sequence of distances $\left\{\rho\left(x_{n+1}, f x_{n}\right)\right\}_{n=1}^{\infty}$ is small in some sense. For the case of round off errors in computer modelling, such a sequence is uniformly small, i.e. there exists some $\varepsilon>0$, such that $\sup _{n} \rho\left(x_{n+1}, f x_{n}\right)<\varepsilon$. The sequence $\bar{x}$ in this case is called an $\varepsilon$-trajectory of the dynamical system $f$ [1]. In a series of investigations [1-14] a study was made of the properties and applications of $\varepsilon$-trajectories.

Dynamical systems with stochastic behaviour always have a large set of invariant measures. However under the presence of a stochastic attractor (the definition is given in $\S 1$ ), which is the type of system we shall consider, there exists a special invariant measure $\mu$, such that each smooth probability distribution on the phase space converges to this measure under the action of the dynamical system. In applied investigations very often this measure is calculated by the histogram of a computer trajectory, i.e. by an $\varepsilon$-trajectory. It is well known that there are not only $\varepsilon$ trajectories, but even true trajectories of dynamical systems with stochastic attractors, the statistics of which essentially differs from the measure $\mu[18,19]$. In $\S 2$ of this paper we show, that in the typical situation the statistics, obtained from an $\varepsilon$ trajectory, 'with probability 1 ' is close to the measure $\mu$. 
A very important and often used result is the fact, that in the case of smooth hyperbolic dynamical systems each $\varepsilon$-trajectory is uniformly traced by some trajectory of the system. In $\$ 3$ we are going to find new sufficient conditions under which this property is valid for a class of piecewise smooth systems.

In the case when distances $\rho\left(x_{n+1}, f x_{n}\right)$ are small only in average (impulse perturbations, stochastic perturbations of Gauss type and so on) there are no results of the tracing trajectories type in the literature. In $\$ 4$ it is shown that for the case of smooth hyperbolic dynamical systems there is in fact such a tracing property, not uniform, but on average along the trajectory.

The main results of the present paper are the proof of the 'genericity' property of the statistics, calculated on an $\varepsilon$-trajectory of a dynamical system with a stochastic attractor (Theorem 1) and the proof of the tracing on the average property for on average $\varepsilon$-trajectories of hyperbolic dynamical systems (Theorem 4). The last result is a generalization of the well-known result of D. V. Anosov about $\varepsilon$-trajectories in the case of perturbations which are small only in average.

\section{Main definitions and assumptions}

Let $(X, \rho)$ be a $d$-dimensional compact manifold (for example the unit cube or the torus with the Eucledian norm), on which a nonsingular (with respect to the Lebesgue measure $m$ on $X$ ) map $f: X \rightarrow X$ is given. Iterations $f^{n}, n=1,2, \ldots$, of the map $f$ generate on $X$ a discrete time dynamical system, which we also denote by $f$.

Following Sinai [3] we shall say that the dynamical system $f$ has a stochastic attractor $\Lambda$ with an $f$-invariant measure $\mu$, if

(a) $\operatorname{supp} \mu=\Lambda$ and any probability absolutely continuous (with respect to the Lebesgue measure on $X$ ) measure with support in a neighbourhood of $\Lambda$ under the action of the dynamical system $f$ converges weakly to $\mu$;

(b) The dynamical system $f \mid \Lambda$ with the measure $\mu$ is mixing.

Further we shall assume for simplicity that the condition (a) in the definition above is fulfilled for any absolutely continuous probability measure on $X$.

Up to the present, among all dynamical systems with stochastic attractors, smooth ones with hyperbolic structure have been the most deeply studied [1-5]. Let us give a definition.

A compact $f$-invariant set $\Lambda$ is called hyperbolic, if the restriction of the tangent bundle of the diffeomorphism $f$ on $\Lambda$ decomposes into a continuous Whitney sum of two sub-bundles $W^{s}(x)$ (stable manifold) and $W^{u}(x)$ (unstable one). Here

$$
\begin{aligned}
& W^{s}(x)=\left\{y \in X: \rho\left(f^{n} x, f^{n} y\right) \rightarrow 0 \text { as } n \rightarrow \infty\right\}, \\
& W^{u}(x)=\left\{y \in X: \rho\left(f^{-n} x, f^{-n} y\right) \rightarrow 0 \text { as } n \rightarrow \infty\right\}, \\
& W_{\varepsilon}^{s}(x)=\left\{y \in X: \rho\left(f^{n} x, f^{n} y\right)<\varepsilon \text { for } n \in \mathbb{Z}_{+}\right\}, \\
& W_{\varepsilon}^{u}(x)=\left\{y \in X: \rho\left(f^{-n} x, f^{-n} y\right)<\varepsilon \text { for } n \in \mathbb{Z}_{+}\right\} .
\end{aligned}
$$

The last two definitions concern local stable and unstable manifolds.

Statement 1.1. (Local direct product structure) [1, 4]. For the hyperbolic dynamical system $f$ there exist constants $0<\varepsilon_{0}<1<\lambda, C_{1}, \gamma<\infty$, such that for all $0<\varepsilon \leq \varepsilon_{0}$. 
(a) for any $x, y \in X$ there exists a point $r(x, y)$ such that

$$
r(x, y) \in W_{\varepsilon}^{u}(x) \cap W_{\varepsilon}^{s}(y), \quad \rho(x, r(x, y))+\rho(y, r(x, y)) \leq C_{1} \rho(x, y) ;
$$

(b) for any $x \in W_{\varepsilon}^{s}(y), y \in X$

$$
\rho\left(f^{n} x, f^{n} y\right) \leq \lambda^{-n} \rho(x, y),
$$

(c) for any $x, y \in \Lambda$

$$
\rho\left(f^{n} x, f^{n} y\right) \leq \gamma^{n} \rho(x, y) .
$$

It is on this statement that the application of so-called Markov partitions of dynamical systems is based [2-4]. Let us call by a rectangle a subset $\Lambda_{i}$ of the attractor, decomposed into local layers of stable $\left(W^{s}\right)$ manifolds and unstable $\left(W^{u}\right)$ ones. By Statement 1.1 the intersection of any local layers consists just of one point. A set of rectangles $\left\{\Lambda_{1}, \ldots, \Lambda_{N}\right\}$, intersecting only by boundaries, is called a Markov partition of the attractor $\Lambda$, if for each internal local layer $W_{\varepsilon}^{u} \subset \Lambda_{i}$ the intersection $f W_{\varepsilon}^{u} \cap \Lambda_{j}$ consists of one or several local layers of unstable manifold lying inside $\Lambda_{j}$ for any $i, j=1,2, \ldots, N$, and (analogously) if $f W_{\varepsilon}^{s}$ lies inside $\Lambda_{j}$, then it is a subset of some local stable layer.

There exist Markov partitions of Anosov systems [1] and of smooth hyperbolic dynamical systems with Axiom A of Smale [4,5]. Partitions of the Markov type are known also for the Lorenz model [3] with discrete time. In the last case there exist stable and unstable manifolds of this system, but because of the discontinuity of the Poincare map $f$, there is no possibility to represent the tangent bundle in the form of their continuous Whitney sum.

For the description of piecewise differentiable dynamical systems of the Lorenz type and systems with not one-to-one mappings $f$, we introduce a class of systems $P C^{1+\alpha}, 0<\alpha<1[18]$. We shall say that $f \in P C^{1+\alpha}$, if there exists a partition of the phase space $X$ intersecting only by boundaries nonempty subsets $X_{i}$ with piecewise differentiable boundaries, such that for all $i$ the map $f_{i}=f \mid$ int $X_{i}$ is a $C^{1}$. diffeomorphism, and the function $|\operatorname{det} D f x|^{-1}$ satisfies on int $X_{i}$ the Holder condition with an exponent $\alpha$ and may be extended as a function, satisfying this condition to $\operatorname{clos} \boldsymbol{X}_{i}$.

The dynamical system $f$ is said to be piecewise expanding, if $f \in P C^{1+\alpha}$ and there exists $\lambda>1$, such that for all $i$ and $x, y \in X_{i}$ the following inequality holds:

$$
\rho(f x, f y)>\lambda \rho(x, y) .
$$

For a survey of properties of dynamical systems of class $P C^{1+\alpha}$, see [18].

Now let us consider the space of sequences $X^{\infty}$ with elements in the phase space $X$ of the dynamical system $f$. The Lebesgue measure $m$ on $X$ induces in the standard manner the measure $m^{\infty}$ on $X^{\infty}$. A sequence $\bar{x} \in X^{\infty}$ is called an $\varepsilon$-trajectory [1] of the map $f$, if $\rho\left(x_{n+1}, f x_{n}\right) \leq \varepsilon$ for all $n \in \mathbb{Z}_{+}$. We introduce also the notion of an $\varepsilon$-a-trajectory, i.e. an average $\varepsilon$-trajectory. A sequence $\bar{x} \in X^{\infty}$ is called an $\varepsilon-$ $a$-trajectory if there exists a number $N=N(\varepsilon)$, such that for all $m>N, k \in \mathbb{Z}_{+}$

$$
m^{-1} \sum_{n=1}^{m} \rho\left(x_{n+k+1}, f x_{n+k}\right) \leq \varepsilon
$$


The $\varepsilon$ - and $\varepsilon$ - $a$-trajectories can be interpreted as trajectories of perturbed systems. In this case to the $\varepsilon$-trajectory we assign perturbations uniformly bounded by a small constant $\varepsilon$ (for example round off errors in computer modelling), and to the $\varepsilon-a$-trajectory - perturbations which are small only on average (for example the realizations of a Gaussian process with zero mean value, impulsive perturbations and so on).

An important example of problems, in which the $\varepsilon-a$-trajectories arise naturally, is the investigation of the most probable paths of discrete time dynamical systems with general Markov perturbations that was carried out in another author's paper.

The investigation of properties of $\varepsilon$-trajectories of dynamical systems was the subject of a series of papers $[6-14,18]$. The most significant result in this field is D. V. Anosov's theorem about $\varepsilon$-trajectories, by which for any $\sigma>0$ one can find $\varepsilon>0$ such that for each $\varepsilon$-trajectory $\bar{x} \in X^{\infty}$ of a smooth hyperbolic dynamical system $f$, there exists a point $x \in X$, such that

$$
\sup _{n} \rho\left(x_{n}, f^{n} x\right) \leq \sigma \text {. }
$$

The last property is called the tracing property of the $\varepsilon$-trajectory. In part 4 of the present paper this result is generalized to the case of $\varepsilon-a$-trajectories, i.e. perturbations, which are small only on average.

For proofs of some statements that we shall need further let us give some properties of functions with bounded $\alpha, \beta$-variation, introduced in [18]. Fix $\alpha, \beta \in(0,1)$ and for equivalence classes in the space of $m$-integrable functions $L^{1}(X, m)$, define the $\alpha, \beta$-variation on a Borel set $Y \subseteq X$ :

$$
V(g, Y)=\inf _{\tilde{g} \in g} \sup _{0<t<\beta} t^{-\alpha} \theta(\tilde{g}, Y, t)
$$

where $\tilde{g}$ is a representative of the equivalence class $g \in L^{1}(X, m)$,

$$
\begin{gathered}
\theta(\tilde{g}, Y, t)=\int_{Y} \underset{B_{r}(x) \cap Y}{\operatorname{Osc}}(\tilde{g}) d x, \quad \operatorname{Osc}_{A}(\tilde{g})=\sup _{A}(\tilde{g})-\inf _{A}(\tilde{g}), \\
B_{t}(A)=\{y \in X: \rho(y, A) \leq t\}-\text { the } t \text {-neighbourhood of the set } A .
\end{gathered}
$$

We denote by $B V(X, \alpha, \beta)$ the space of functions with bounded $\alpha, \beta$-variation from $L^{1}(X, m)$. As was shown in [18], this space, equipped with the norm

$$
\|g\|_{V}=V(g, X)+\|g\| \text {, }
$$

becomes a Banach space. We shall say that a function $g \in L^{1}(X, m)$ satisfies some property, if there exists a representative of the same equivalence class which satisfies this property.

Statement 1.2 [18] (a) If $\left\{g_{n}\right\}_{n=1}^{\infty} \subset B V(X, \alpha, \beta),\left\|g_{n}\right\| \leq M<\infty$ and there exists $g \in L^{1}(X, m)$ such that $\left\|g_{n}-g\right\| \rightarrow 0$ as $n \rightarrow \infty$, then $g \in B V(X, \alpha, \beta)$ and $\|g\|_{V} \leq M$;

(b) each bounded in $B V(X, \alpha, \beta)$ set is a compact set in $L^{1}(X, m)$;

(c) if $\left\{g_{n}\right\}_{n=1}^{\infty} \subset B V(X, \alpha, \beta)$ then for any Borel set $Y \subseteq X$

$$
V\left(\sum_{n=1}^{N} g_{n}, Y\right) \leq \sum_{n=1}^{N} V\left(g_{n}, Y\right)
$$

(d) if $g \in B V(X, \alpha, \beta)$ and $X \subset R^{d}$, then

$$
\|g\|_{\infty} \leq(2 d / \beta)^{d}\|g\|_{v} .
$$


We shall also need some facts from the measure theory. Denote by $M(X)$ the set of normalized Borel measures on $X$. A sequence of measures $\left\{\mu_{n}\right\}_{n=1}^{\infty} \subset M(X)$ is called weakly converging to a measure $\mu \in M(X)$ if for any continuous on $X$ function $h$,

$$
\int h d \mu_{n} \rightarrow \int h d \mu \quad \text { as } n \rightarrow \infty
$$

In $M(X)$ one can introduce a metric $\rho_{*}(\cdot, \cdot)$ which is equivalent to the weak convergence of measures. For this purpose, in the space of continuous functions on $X$ with the uniform norm $\|\cdot\|_{C}$, we fix a dense family of functions $\left\{h_{n}\right\}_{n=1}^{\infty}$ and define this metric by the following formula

$$
\rho_{*}\left(\mu_{1}, \mu_{2}\right)=\sum_{n=1}^{\infty} 2^{-n}\left\|h_{n}\right\|_{C}^{-1}\left|\int h_{n} d \mu_{1}-\int h_{n} d \mu_{2}\right| .
$$

\section{Statistical properties of the $\varepsilon$-trajectories}

In this section we shall investigate typical statistical properties of $\varepsilon$-trajectories of dynamical systems with stochastic attractors. Let $\bar{x} \in X^{\infty}$ be a trajectory of the dynamical system $f$ and $\bar{x}^{(\varepsilon)} \in X^{\infty}$ be an $\varepsilon$-trajectory. Denote

$$
\begin{gathered}
\mu\left(\bar{x}^{(\varepsilon)}, N\right)=N^{-1} \sum_{n=1}^{N} \delta\left(x_{n}^{(\varepsilon)}\right), \\
\mu(\bar{x}, N)=N^{-1} \sum_{n=1}^{N} \delta\left(x_{n}\right),
\end{gathered}
$$

where $\delta(x)$ is a $\delta$-function on $X$, concentrated at the point $x \in X, \bar{x}=\left\{x_{n}\right\}_{n=1}^{\infty}$, $\bar{x}^{(\varepsilon)}=\left\{x_{n}^{(\varepsilon)}\right\}_{n=1}^{\infty}$.

In view of the compactness of the phase space $X$ of the dynamical system $f$, the sequences of measures $\{\mu(\bar{x}, N)\}_{N=1}^{\infty},\left\{\mu\left(\bar{x}^{(\varepsilon)}, N\right)\right\}$ have limit points $\mu(\bar{x})$ and $\mu\left(\bar{x}^{(\varepsilon)}\right)$ respectively.

A question about the connection between the measure $\mu(\bar{x})$ and the measure $\mu$ on the stochastic attractor $\Lambda$ of the dynamical system was studied in a series of papers $[6,7,13,18]$. A trajectory $\bar{x}$ of the dynamical system $f$ is called typical if $\mu(\bar{x})=\mu$. From the Birkhoff egodic theorem it follows that for $\mu$-almost all points $x \in X$ the genericity property is fulfilled. More delicate properties of the set of typical points of dynamical systems were studied for example in $[4,6,7]$.

We shall be interested in an analogous problem for the limit measures $\mu\left(\bar{x}^{(\varepsilon)}\right)$, constructed not along the trajectory, but along the $\varepsilon$-trajectory of the dynamical system. As it is easy to see, an $\varepsilon$-trajectory, in distinction to a true trajectory of the system, is not defined already by its initial point. Therefore the notion of the genericity here has to be defined for elements $\bar{x}^{(\varepsilon)}$ of the infinite dimensional space $\left(X^{\infty}, m^{\infty}\right)$. Fix $\sigma_{0}>0$.

We shall call an $\varepsilon$-trajectory $\bar{x}^{(\varepsilon)} \sigma$-typical if there exists a natural number $N_{\sigma}$, such that for all $N>N_{\sigma}$,

$$
\rho_{*}\left(\mu\left(\bar{x}^{(\varepsilon)}, N\right), \mu\right)<\sigma<\sigma_{0}
$$


THEOREM 1. Let a dynamical system $f \in P C^{1+\alpha}$, inf $\mid$ det $D f x \mid>0$ and there exists a globally attracting stochastic attractor $(\Lambda, \mu)$. Then for any $0<\sigma<\sigma_{0}$ there exists $\varepsilon>0$, such that for $m^{\infty}$-almost all $\varepsilon$-trajectories of the dynamical system are $\sigma$-typical.

COROLlary 1. For sufficiently small $\varepsilon>0$ there exists $N_{0}$ such that for all $N>N_{0}$ measures $\mu\left(\bar{x}^{(\varepsilon)}, N\right)$, determined by $m^{\infty}$-almost all $\varepsilon$-trajectories, are close to the f-invariant measure $\mu$ on the stochastic attractor.

COROLlaRy 2. If we additionally assume that the dynamical system $f$ possesses the tracing property, then for sufficiently small $\varepsilon>0$ for $m^{\infty}$-almost all $\varepsilon$-trajectories, the trajectories of the dynamical system tracing these $\varepsilon$-trajectories are typical.

For the proof of this theorem we need some results about small random perturbations of dynamical systems with stochastic attractors.

Let us fix a family of stochastic Markov processes $\left\{\pi_{\varepsilon}\right\}_{\varepsilon>0}$ on the phase space $X$ of the dynamical system under consideration. This family is defined by its transition probability densities

$$
\begin{array}{ll}
q_{\varepsilon}(x, y)=1 / m\left(B_{\varepsilon}(x) \cap X\right) & \text { for } y \in B_{\varepsilon}(x) \cap X, \\
q_{\varepsilon}(x, y)=0 & \text { for } y \notin B_{\varepsilon}(x) \cap X .
\end{array}
$$

The Markov process $f_{e}$, which is equal to the superposition of the initial dynamical system $f$ and the stochastic process $\pi_{\varepsilon}$, is called a stochastically $\varepsilon$-perturbed system. In other words under the action of $f_{\varepsilon}$ a point $x \in X$ at first transforms to a point $f x$ and then to a random point $y$, which is chosen according to the transition probability density $q_{\varepsilon}(f x, y)$.

As it is easy to see, each realization of the Markov process $f$ is an $\varepsilon$-trajectory of the initial system, that allows us to apply results about small random perturbations of dynamical systems for the investigation of properties of $\varepsilon$-trajectories.

LEMMA 2.1. The random process $f_{\varepsilon}$ possesses a unique normalized invariant measure $\mu_{\varepsilon}$ which is absolutely continuous with respect to Lebesgue measure.

Proof. The dynamical system $f$ induces in the space of probability densities the transformation $P$, which is called the Perron-Frobenius operator and is defined by the relation

$$
\int_{A} P h(x) d x=\int_{f^{-1} A} h(x) d x, \quad h \in L^{1}(X, m)
$$

for any Borel set $A \subseteq X$. An analogous operator $Q_{\varepsilon}$ for the Markov process $\pi_{\varepsilon}$ is defined by the relation

$$
Q_{\varepsilon} h(x)=\int h(x) q_{\varepsilon}(u, x) d u, \quad h \in L^{1}(X, m) .
$$

By definition of the stochastically perturbed system, the operator, describing the dynamics of probability densities under the action of $f_{\varepsilon}$, is represented in the form $P_{\varepsilon}=Q_{\varepsilon} P$.

Let us fix $\varepsilon>0$ and show, that there exists $\gamma_{\varepsilon}<\infty$, such that for any function $h \in B V(X, \alpha, \beta)$ the following inequality holds:

$$
V\left(Q_{\varepsilon} h, X\right)<\gamma_{\varepsilon}\|h\| .
$$


Really, for any $h \in B V(X, \alpha, \beta)$

$$
\operatorname{Osc}(h)<\sup _{|w| \leq 1} \int_{A} h(x) w(x) d x,
$$

where $w \in L^{1}(X, m),|\cdot|$ is the Euclidean norm. From this

$$
\begin{aligned}
V\left(Q_{\varepsilon} h, X\right) & <\sup _{0<t<\beta} t^{-\alpha} \int_{X} \underset{B_{t}(x) \cap X}{\operatorname{Osc}}\left(Q_{\varepsilon} h\right) d x \\
& \leq \sup _{0<t<\beta} t^{-\alpha} \int_{X} \sup _{|w| \leq 1} \int_{B_{t}(x) \cap X} w(s) Q_{\varepsilon} h(s) d s d x \\
& =\sup _{0<t<\beta} t^{-\alpha} \iint_{X}\left[\sup _{|w| \leq 1} \int_{B_{t}(x) \cap X} w(s) q_{\varepsilon}(u, s) d s\right] h(u) d u d x \\
& \leq \sup _{0<t<\beta} t^{-\alpha} \iint_{X}\left[\sup _{x, y} q_{\varepsilon}(x, y)\right] \sup _{|w| \leq 1} \int_{B_{t}(x) \cap X}|w(s)| d s|h(u)| d u d x \\
& \leq C \sup _{0<t<\beta} t^{-\alpha} m^{-1}\left(B_{\varepsilon}(0)\right)\left[\int_{X}|h(u)| d u\right] \int_{X} m\left(B_{t}(x) \cap X\right) d x \\
& \leq C m^{-1}\left(B_{\varepsilon}(0)\right)\|h\| \sup _{0<t<\beta} t^{-\alpha} m\left(B_{t}(0)\right) \\
& =C m^{-1}\left(B_{\varepsilon}(0)\right)\|h\| \beta^{-\alpha} m\left(B_{\beta}(0)\right)
\end{aligned}
$$

because $\alpha, \beta$ satisfy the inequality $0<\alpha, \beta<1$. Hence

$$
V\left(P_{\varepsilon} h, X\right)=V\left(Q_{\varepsilon} P h, X\right) \leq \gamma_{\varepsilon}\|P h\| \leq \gamma_{\varepsilon}\|h\| .
$$

Now we show that for each function $h \in L^{1}(X, m)$

Really,

$$
N^{-1} \sum_{n=1}^{N} P_{\varepsilon}^{n} h \stackrel{L^{1}}{\longrightarrow} h_{\varepsilon} \in B V(X, \alpha, \beta) \quad \text { as } N \rightarrow \infty
$$

$$
V\left(N^{-1} \sum_{n=1}^{N} P_{\varepsilon}^{n} h, X\right) \leq N^{-1} \sum_{n=1}^{N} V\left(P_{\varepsilon}^{n} h, X\right) \leq \gamma_{\varepsilon}\|h\| .
$$

Therefore, by the Property 1 2(b) of functions with bounded $\alpha, \beta$-variation the sequence $\left\{N^{-1} \sum_{n=1}^{N} P_{\varepsilon}^{n} h\right\}$ has a limit point $h_{\varepsilon}$, which is a function of bounded $\alpha, \beta$-variation. Applying the statistical ergodic theorem [20], we obtain that this sequence converges to $h_{\varepsilon}$ also in the $L^{1}$-norm and $P_{\varepsilon} h_{\varepsilon}=h_{\varepsilon}$. That proves the fact that the measure $\mu_{\varepsilon}$ with the density $h_{\varepsilon}$ is the $f_{\varepsilon}$-invariant measure.

For the proof of uniqueness of this measure it is enough to check up the fulfilling of the Doeblin condition [20]. Let $\mathscr{P}_{\varepsilon}(x, A)$ be the probability of a transition of the Markov process $f_{\varepsilon}$ from a point $x \in X$ to a Borel set $A \subseteq X$. Then

$$
\mathscr{P}_{\varepsilon}(x, A)=\int_{A} q_{\varepsilon}(f x, y) d y \leq C m^{-1}\left(B_{\varepsilon}(0)\right) \int_{A} d y \leq \gamma_{\varepsilon} m(A) .
$$

From which, if we fix $\delta=\gamma_{\varepsilon}^{-2}$, then for any Borel set $A$ with Lebesgue measure $m(A)<\delta$, the Doeblin condition is fulfilled.

Statement 2.1. Under the conditions of the theorem above the measures $\mu_{\varepsilon} \rightarrow \mu$ as $\varepsilon \rightarrow 0$. 
Under different assumptions about the dynamical system $f$ and its perturbations this statement was proven in papers $[13-16,18]$.

Leмma 2.2. For $m^{\infty}$-almost all realizations $\bar{x}^{(\varepsilon)} \in X^{\infty}$ of the random process $f_{\varepsilon}$ the measures $\mu\left(\bar{x}^{(\varepsilon)}, N\right) \rightarrow \mu_{\varepsilon}$ as $N \rightarrow \infty$.

This statement is a consequence of the Doeblin condition for the random process $f_{\varepsilon}$.

Now we are ready to prove the theorem. By Statement 2.1 , for any $\sigma>0$ there exists $\varepsilon>0$, such that $\rho_{*}\left(\mu_{\varepsilon}, \mu\right)<\sigma$. On the other hand, by Lemma 2.2 it follows that for $m^{\infty}$-almost all realizations $\bar{x}^{(\varepsilon)}$ of the random process $f_{\varepsilon}$ (i.e. $\varepsilon$-trajectories of the system $f)$ the limit measures $\mu\left(\bar{x}^{(\varepsilon)}\right)$ coincide with the measure $\mu_{\varepsilon}$. Hence $\rho_{*}\left(\mu\left(\bar{x}^{(\varepsilon)}\right), \mu\right)<\sigma$ for $m^{\infty}$-almost relizations of $f_{\varepsilon}$.

Corollary 1 is equivalent to the theorem and Corollary 2 follows from the fact that a trajectory $\bar{x}$ of the dynamical system $f$, tracing an $\varepsilon$-trajectory $\bar{x}^{(\varepsilon)}$ lies uniformly close to it.

\section{Tracing conditions for $\varepsilon$-trajectories}

For the first time the tracing property for $\varepsilon$-trajectories was discovered in paper [1] for the case of smooth hyperbolic dynamical systems. From that time a series of papers $[6-14,18]$ was dedicated to the investigation of this property and to its applications to both theoretical and applied problems. However, there has been no increase of the class of dynamical systems for which the tracing property is proven. The point is the fact that the proof of the Anosov theorem about the tracing of $\varepsilon$-trajectories essentially uses the existence and properties of smooth stable and unstable manifolds of the hyperbolic dynamical systems. Therefore to extend this result to a class of piecewise continuous dynamical systems, possessing for example critical points, we have to use sufficiently different techniques of proofs. In the present section we shall obtain some sufficient conditions for the validity of the tracing property for such systems.

THEOREM 2. Let $f$ be a piecewise expanding map with expanding constant $\lambda>1$ and

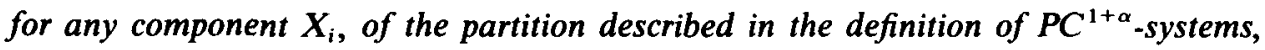
assume the condition $f X_{i}=X$ is valid. Then for any $\sigma>0$ there exists $\varepsilon>0$ such that any $\varepsilon$-trajectory of the system $f$ is uniformly traced by a trajectory of this system with the accuracy $\sigma$.

Proof. Let us denote $Y=\bigcup_{i} \partial X, \hat{Y}=\bigcup_{n=0}^{\infty} f^{-n} Y, \hat{X}=X \backslash \hat{Y}$. By the construction of the set $\hat{X}$, it is a dense set in $X$ and for any $i$ it fulfils $f\left(\hat{X} \cap X_{i}\right)=\hat{X}$. For any point $x \in X_{i}$ we denote by $X(x)$ the set $X_{i}$.

Fix $\varepsilon>0$ small enough. Then in view of the expanding property, we have for any $x, y \in X$, such that $\rho(f x, y)<\varepsilon$ that there exists a point $y^{\prime} \in X(x) \cap \hat{X}$ with properties $f y^{\prime}=y$ and

$$
\rho\left(x, y^{\prime}\right)<\varepsilon / \lambda .
$$

Really, for example, let $x \in X_{i}$. By the expanding condition, setting $y^{\prime}=f_{i}^{-1} y$, we 
obtain

$$
\varepsilon>\rho(y, f x)=\rho\left(f f_{i}^{-1} y, f f_{i}^{-1} f x\right) \geq \lambda \rho\left(f_{i}^{-1} y, f_{i}^{-1} f x\right)=\lambda \rho\left(y^{\prime}, x\right) .
$$

Now choose $\sigma \in(0, \varepsilon /(1-1 / \lambda))$. Suppose that an $\varepsilon$-trajectory $\bar{x}^{(\varepsilon)}=\left\{x_{n}^{(\varepsilon)}\right\}_{n=1}^{\infty} \subset \hat{X}$ (in the general case in view of the fact that $X$ is a dense set in $X$ it is enough to approximate $\bar{x}^{(\varepsilon)}$ by a sufficiently close $\varepsilon$-trajectory $\left.\hat{x}^{(\varepsilon)} \subset \hat{X}\right)$. Choose an arbitrary natural number $N$ and set $y_{N}=x_{N}^{(\varepsilon)}$. As $\rho\left(f x_{N-1}^{(\varepsilon)}, y_{N}\right) \leq \varepsilon$, then there exists $y_{N-1} \in$ $X\left(x_{N-1}^{(\varepsilon)}\right) \cap \hat{X}$ such that

$$
\rho\left(x_{N-1}^{(\varepsilon)}, y_{N-1}\right) \leq \varepsilon \lambda^{-1}, \quad f y_{N-1}=y_{N} .
$$

Now since

$$
\rho\left(f x_{N-2}^{(\varepsilon)}, y_{N-1}\right)<\rho\left(f x_{N-2}^{(\varepsilon)}, x_{N-1}^{(\varepsilon)}\right)+\rho\left(x_{N-1}^{(\varepsilon)}, y_{N-1}\right) \leq \varepsilon(1-1 / \lambda)<\sigma,
$$

then there exists $y_{N-2} \in X\left(x_{N-2}^{(\varepsilon)}\right) \cap \hat{X}$ such that $f y_{N-2}=y_{N-1}$ and

$$
\rho\left(x_{N-2}^{(\varepsilon)}, y_{N-1}\right)<\varepsilon\left(1+\lambda^{-1}+\lambda^{-2}\right) .
$$

Continuing this construction, we have for arbitrary $n \leq N$, that there exists $y_{n} \in$ $X\left(x_{n}^{(\varepsilon)}\right) \cap \hat{X}$, such that $f y_{n}=y_{n+1}$ and

$$
\rho\left(x_{n}^{(\varepsilon)}, y_{n}\right) \leq \varepsilon\left(1+\lambda^{-1}+\lambda^{-2}+\cdots\right)<\varepsilon /(1-1 / \lambda)<\sigma .
$$

In such a way we construct the segment of the trajectory $\left\{y_{n}\right\}$ of the dynamical system $f$, uniformly tracing the $\varepsilon$-trajectory $\bar{x}^{(\varepsilon)}$ on the time interval $[0, N]$. But since the number $N$ can be chosen arbitrarily large, then the statement of the theorem follows.

We remark that for the one-dimensional representation of the Lorenz system a close result was obtained earlier in the paper [8].

It turns out that the condition $f X_{i}=X$ of Theorem 2 is in a sense a necessary one for the tracing property of $\varepsilon$-trajectories which follows from the results of papers $[8,11,18]$.

As was shown in $[18,22]$ a powerful method for the extension of results about dynamical systems with stochastic behaviour is a topological conjugation of such systems, i.e. the constructing of a system $\hat{f}=h^{-1} f h$ for the dynamical system $f$. Here $h$ is a homeomorphism of the phase space $X$ into itself. The existence conditions of the topological conjugacy for different classes of dynamical systems can be found for example in $[5,17,22]$. A class of dynamical systems topologically conjugated to piecewise expanding or hyperbolic systems is sufficiently more broad than initial ones, for which the tracing property is known. For example the next statement is valid.

LеммA 3.1. Let $f$ be a piecewise expanding or hyperbolic dynamical system. Then there exists a homeomorphism $h: X \rightarrow X$ such that there is a critical point in the system $\hat{f}=h f h^{-1}$.

Proof. By the conditions there exists a point $x \in X$ such that $f x \neq x$ and $f$ is a local $C^{1}$-diffeomorphism in a small neighbourhood of points $x$ and $f x$. If there exist corresponding derivatives the following equality is valid

$$
h^{\prime}(x) \hat{f}^{\prime}(h x)=f^{\prime}(x) h^{\prime}(f x)
$$

Hence each homeomorphism $h: X \rightarrow X$ is locally differentiable at points $x, f x \in X$, 
such that $h^{\prime}(x) \neq 0$ and $h^{\prime}(f x)=0$, conjugates the map $f$ with the map possessing a critical point.

THEOREM 3. Let a dynamical system $f$ be conjugate by means of a homeomorphism $h$ to a system $\hat{f}=h^{-1} f h$, possessing the tracing property. Then $f$ also possesses this property.

Proof. Let $\left\{\hat{x}_{n}\right\}_{n=0}^{\infty} \in X^{\infty}$ be an $\hat{\varepsilon}$-trajectory of the map $f$, i.e. $\rho\left(\hat{f} \hat{x}_{n}, \hat{x}_{n+1}\right) \leq \hat{\varepsilon}$ for all natural $n$. Denote $x_{n}=h^{-1} \hat{x}_{n}$ and consider the sequence $\left\{x_{n}\right\}_{n=0}^{\infty} \in X^{\infty}$. By the definition

$$
\hat{\varepsilon} \geq \rho\left(\hat{x}_{n+1}, \hat{f} \hat{x}_{n}\right)=\rho\left(h x_{n+1}, h h^{-1} \hat{f} h x_{n}\right)=\rho\left(h x_{n+1}, h f x_{n}\right) .
$$

But as $h$ is a homeomorphism, then $h$ is a continuous and hence also uniformly continuous map of the compact set $X$ into itself. Therefore for any $\varepsilon>0$ there exists $\hat{\varepsilon}>0$ such that if $\rho(x, y)<\hat{\varepsilon}$ then $\rho(h x, h y)<\varepsilon$. In such a way the sequence $\left\{x_{n}\right\}_{n=0}^{\infty}$ is an $\varepsilon$-trajectory of the map $f(\varepsilon$ and $\hat{\varepsilon}$ here are connected in a way mentioned above).

As the map $\hat{f}$ possesses the tracing property, then for any $\sigma>0$ there exists $\varepsilon>0$ such that for any $\hat{\varepsilon}$-trajectory $\left\{\hat{x}_{n}\right\}_{n=0}^{\infty}$ of the system $\hat{f}$ there exists a point $\hat{x} \in X$ such that $\rho\left(\hat{f}^{n} \hat{x}, \hat{x}_{n}\right)<\sigma$ for any natural $n$. If we set $x=h \hat{x}$, then

$$
\sigma>\rho\left(\hat{f}^{n} \hat{x}, \hat{x}_{n}\right)=\rho\left(h^{-1} h \hat{f}^{n} h^{-1} h x, h^{-1} h \hat{x}_{n}\right)=\rho\left(h^{-1} f^{n} x, h^{-1} x_{n}\right) .
$$

Now, applying again the uniform continuity of the map $h$, we obtain that the trajectory $\left\{f^{n} x\right\}_{n=0}^{\infty}$ also uniformly traces the $\varepsilon$-trajectory of the map $f$ under consideration.

\section{Properties of $\varepsilon-a$-trajectories}

In this section we shall study properties of trajectories of perturbed hyperbolic dynamical systems, when the perturbations are small only in average, i.e. $\varepsilon-$ $a$-trajectories. We shall restrict ourselves to the case when the map $f$ satisfies the Smale Axiom A on the whole phase space $X$ (i.e. the $w$-limit set of the map $f$ coincides with $X$ and periodic points of $f$ are dense in $X$ ). In the opposite case we can restrict, as is usually done, the dynamical system $f$ to its locally maximal hyperbolic set $[4,5]$.

THEOREM 4. For any $\sigma>0$ there exists $\varepsilon>0$ such that for any $\varepsilon-a$-trajectory $\bar{x} \in X^{\infty}$ there exists a trajectory of the system $f$ tracing on average the trajectory $\bar{x}$ with the accuracy $\sigma$, i.e. there is $x \in X$, such that the following inequality holds:

$$
\varlimsup_{N \rightarrow \infty} N^{-1} \sum_{n=1}^{N} \rho\left(x_{n}, f^{n} x\right) \leq \sigma .
$$

To simplify calculations we shall assume further that $\rho(\cdot, \cdot)$ is the Lyapunov metric [1] for the map $f$ on $X$.

For the proof of this theorem we need some generalization of the Statement 1.1 about the local structure of the direct product in the nonlocal case.

LEMMA 4.1. There are constants $A, B>0$ such that for any pair of points $x, y \in X$ there exists a point $r(x, y) \in X$, such that

(a) $r(x, y) \in W^{u}(x) \cap W^{s}(y)$;

(b) $\rho(r(x, y), x)+\rho(r(x, y), y) \leq A \rho(x, y)$; 
(c) $\rho\left(f^{-n} r(x, y), f^{-n} x\right) \leq B \lambda^{-n} \rho(x, y) \quad$ for $n \geq 0, \lambda>1$;

(d) $\rho\left(f^{n} r(x, y), f^{n} y\right) \leq B \lambda^{-n} \rho(x, y)$ for $n \geq 0, \lambda>1$.

Proof. We shall use the fact, that for the Axiom A hyperbolic dynamical system $f$ there exists a Markov partition whose elements have an arbitrary small diameter $[4,5]$.

Let us fix a Markov partition with a diameter less than the constant $\varepsilon_{0}$ (from Statement 1.1). Because of the transitivity of the map $f$ there is an exponent $n_{0}$, such that the transition matrix of the Markov partition (i.e. the matrix with elements 0 or 1 , assigning to transitions under the action of $f$ from the one element of the partition to another) in this degree is strictly positive. Therefore after the time $\boldsymbol{n}_{0}$ all stable and unstable layers are sure to intersect, i.e. the condition (a) of the theorem is fulfilled and, accordingly there is a point $r(x, y)$ in their intersection. This point may be not unique.

Points $f^{-n_{0}} x$ and $f^{-n_{0}} y$ lie in some elements of our Markov partition. Since after the time $n_{0}$ images of these two elements intersect, then we have an estimate

$$
\rho(x, r(x, y))+\rho(r(x, y), y)<\gamma^{n_{0}} \varepsilon_{0}+\gamma^{n_{0}} \varepsilon_{0}=2 \varepsilon_{0} \gamma^{n_{0}}=A \varepsilon_{0},
$$

where the constant $\gamma$ coincides with the corresponding constant in the Statement 1.1. The item (b) of the lemma is a consequence of this inequality for the case $\rho(x, y) \geq \varepsilon_{0}$ (the case $\rho(x, y)<\varepsilon_{0}$ follows from the Statement 1.1.a).

Now we prove the item (c). Consider an element of the Markov partition containing the point $r(x, y)$. The preimage of this element of degree $n_{0}$ intersects the trajectory of the point $x$ and therefore the point $f^{-n}(r(x, y)) \in W_{\varepsilon_{0}}^{u}(x)$ for $n \geq n_{0}$, and hence by the Statement 1.1.b, item (c) is valid. Part (d) of the lemma is proven analogously.

Proof of Theorem 4. Let $\left\{x_{n}\right\}_{n=1}^{\infty}$ be an $\varepsilon-a$-trajectory of the map $f$. Fix a natural number $k>1$ and choose segments of this sequence so, that for any segment the following inequality is valid

$$
\xi_{m}=\rho\left(x_{m-1}, f x_{m}\right) \leq k \varepsilon .
$$

Denote by $n_{i}$ the length of the $i$ th segment (i.e. the number of points in it). By the theorem about $\varepsilon$-trajectories, for sufficiently small $\varepsilon>0$ for any constructed segment of the sequence there exists a segment of a trajectory of the map $f$, uniformly approximating it with the accuracy $\sigma$. By the construction a distance between the end of the $i$ th segment $\left(x_{i}^{(+)}\right)$and the beginning of the $(i+1)$ th one $\left(x_{i+1}^{(-)}\right) \Delta_{i}$ is less than $\hat{\xi}_{n_{i}}+2 \varepsilon$, where $\hat{\xi}_{n_{i}}=\rho\left(f x_{i}^{(+)}, x_{i+1}^{(-)}\right)$is the distance between the $f$-image of the last point of the $i$ th segment and the first point of the $(i+1)$ th segment.

The approximating on average trajectory of the system $f$ is constructed inductively by steps. At first we approximate the first pair of segments, then the constructed segment of the approximation and the third of initial ones and so on. Here the approximation is a choice of a segment of the trajectory, constructed for the end $x^{(+)}$and the beginning $x^{(-)}$of two consecutive segments by the point $r\left(x^{(+)}, x^{(-)}\right)$. The existence of such an approximation and its properties were described in Lemma 4.1. 


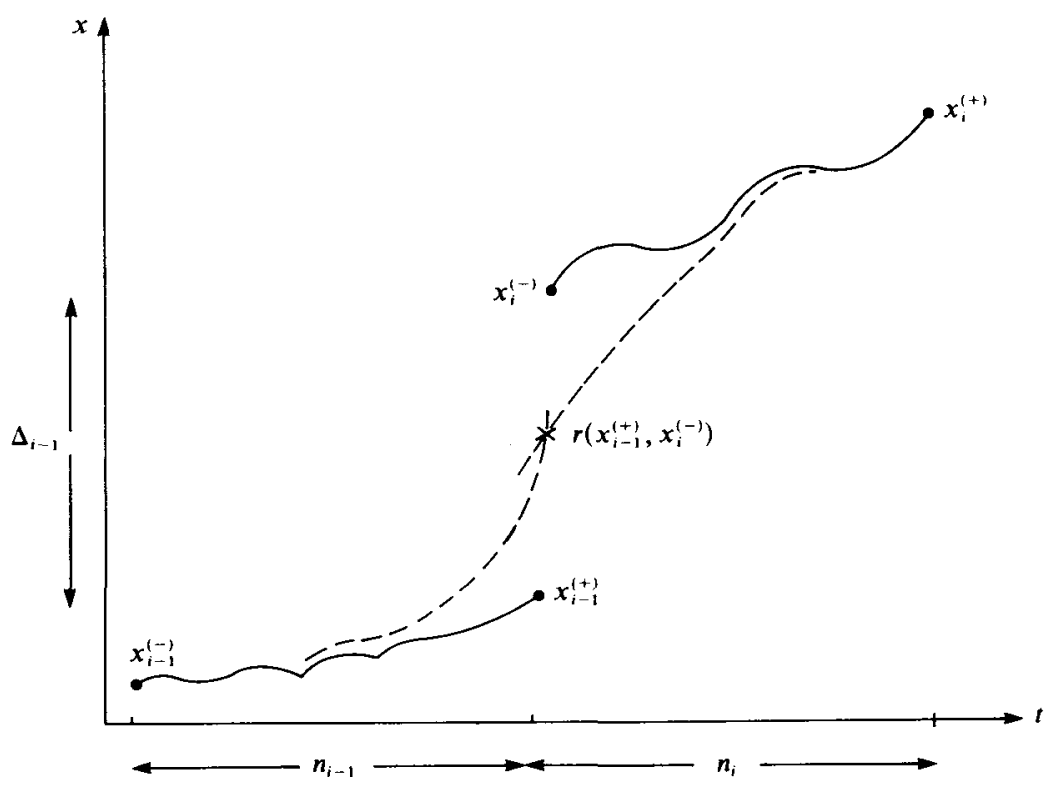

Denote by $\Delta_{i}^{\prime}$ the distance between the last point of the approximation, constructed at the $i$ th step of the procedure and the first point of the next segment $\left(\Delta_{1}^{\prime}=\Delta_{1}\right)$, and by $S_{i}$ the sum of distances from points of segments of trajectories to the approximating trajectory also to the $i$ th step of the procedure $\left(S_{1}=0\right)$.

From the estimates of Lemma 4.1 there follows:

$$
\begin{aligned}
& \Delta_{i+1}^{\prime} \leq B \lambda^{-n_{i+1}} \Delta_{i}^{\prime}+\Delta_{i+1} \\
& S_{i+1} \leq S_{i}+2 B(1-1 / \lambda)^{-1} \Delta_{i}^{\prime} .
\end{aligned}
$$

Denoting by $\alpha_{i}=B \lambda^{-n_{i+1}}, \beta=2 B /(1-1 / \lambda)$, we obtain

$$
\begin{aligned}
S_{i+1} \leq & \beta\left[\Delta_{1}\left(1+\alpha_{1}+\alpha_{1} \alpha_{2}+\cdots+\alpha_{1} \alpha_{2} \cdots \cdots \alpha_{i}\right)\right. \\
& \left.+\Delta_{2}\left(1+\alpha_{2}+\alpha_{2} \alpha_{3}+\cdots+\alpha_{2} \alpha_{3} \cdots \alpha_{i}\right)+\cdots+\Delta_{i}\right] .
\end{aligned}
$$

LEMMA 4.2. There are constants $0<q<1, k \in \mathbb{Z}_{+}$such that for any $m>N$ (from the definition of the $\varepsilon$-a-trajectory) and natural $M$ the following inequality holds

$$
\prod_{i=1}^{m} \alpha_{i+M}<q^{m}
$$

Proof. By the definition of quantities $\alpha_{i}$

$$
\begin{aligned}
Q_{m}^{(M)}=\prod_{i=1}^{m} \alpha_{i+M} & =B^{m} \lambda^{n_{M}+\cdots+n_{M+m-1}} \\
& =\left(B \lambda^{-k}\right)^{m} \exp \left(-m\left(m^{-1} \sum_{i=0}^{m-1} n_{i+M-k}\right) \ln \lambda\right) .
\end{aligned}
$$

On the other hand by the definition of an $\varepsilon-a$-trajectory and its segments it follows 
that

$$
m^{-1} \sum_{i=0}^{m-1} n_{i+M}>k
$$

as for $L=\sum_{i=0}^{m-1} n_{i+M}$ the next inequality is valid

$$
L^{-1} \sum_{i=1}^{L} \rho\left(x_{i+M-1}, f x_{i+M}\right) \leq \varepsilon .
$$

Leaving in the last inequality only terms exceeding the value $k$, we obtain an estimate

$$
L^{-1} m k \sigma<L^{-1} \sum_{i=1}^{L} \rho\left(x_{i+M-1}, f x_{i+M}\right) \leq \varepsilon,
$$

i.e. $L / m \geq k$ or $m^{-1} \sum_{i=0}^{m-1} n_{i+M}>k$.

In such a way $Q_{m}^{(M)} \leq\left(B \lambda^{-k}\right)^{m}$, as $\lambda>1$. Now choosing a natural number $k$ such that

$$
q=B \lambda^{-k}<1
$$

we obtain the desired estimate.

Continuation of the proof of the theorem. By Lemma 4.2

$$
\begin{aligned}
Q_{i} & =1+\alpha_{i}+\alpha_{i} \alpha_{i+1}+\cdots+\alpha_{i} \alpha_{i+1} \cdots \cdots \alpha_{i+j}+\cdots \\
& \leq 1+\alpha_{i}+\cdots+\alpha_{i} \alpha_{i+1} \cdots \cdots \alpha_{i+M}+1 /\left(1-q^{M}\right) \\
& \leq M \lambda^{-M} B^{M}+1 /\left(1-q^{M}\right)=\hat{q}<\infty
\end{aligned}
$$

and this estimate does not depend on the number $i$. So

$$
S_{i+1}<\beta\left[\Delta_{1} Q_{1}+\Delta_{2} Q_{2}+\cdots+\Delta_{i} Q_{i}\right] \leq \beta \hat{q} \sum_{j=1}^{i} \Delta_{j}
$$

Hence

$$
\begin{aligned}
N^{-1} \sum_{n=1}^{N} \rho\left(x_{n+1}, f^{n} x\right) & \leq \beta \hat{q}\left(N^{-1} \sum_{n=1}^{N} \rho\left(x_{n+1}, f x_{n}\right)+2 \varepsilon\right)+\varepsilon \\
& \leq 4 \varepsilon \beta \hat{q},
\end{aligned}
$$

which finishes the proof of the theorem.

Lemma 4.2 shows that in a general case the approximation parameter $k$ exceeds 1 and, hence, we could not inductively approximate the $\varepsilon-a$-trajectory by segments of trajectories of the initial system.

\section{REFERENCES}

[1] D. V. Anosov. Geodesic flows on closed Riemannian manifolds with negative curvature. Tr. Institute of math. AN SSSR 90 (1967).

[2] Y. G. Sinai. Gibbs measures in ergodic theory. Uspekhi Mat. Nauk 27(4) (1972), 21-64.

[3] Y. G. Sinai. Stochasticity of dynamical systems. In: Nonlinear Waves Nauka: Moscow, 1979.

[4] R. Bowen. Methods of Symbolic Dynamics Mir: Moscow, 1979.

[5] Z. Nitecki. Introduction to Differential Dynamics Mir: Moscow, 1975.

[6] K. Sigmund. Generic properties of invariant measures for Axiom A diffeomorphisms. Inventiones Math. 11 (1970), 99-109. 
[7] K. Sigmund. On dynamical systems with the specification property. Trans. Amer. Math. Soc. 190 (1978), 285-299.

[8] M. Komuro. Lorenz attractors do not have the pseudo-orbit tracing property. J. Math. Soc. Jap. 37(3) (1985), 479-514.

[9] K. Kato. Pseudo-orbit and stability of flows. Mem. Fac. Sci. Kochi Univ. (Math.) 5 (1984), 49-62.

[10] K. Kato \& A. Morimoto. Topological $\Omega$ stability of Axiom A flows with no $\Omega$-explosions. $J$. Diff. Eq. 34 (1979), 464-481.

[11] A. Morimoto. The method of pseudo-orbit tracing and stability of dynamical systems. Seminar Note, 1979, 39.

[12] P. Walters. On pseudo-orbit tracing property and its relationship to stability. Lect. Notes Math. 668 (1978), 291-294.

[13] A. Boyarsky. On the significance of absolutely continuous invariant measures. Physica 11D (1984), $130-146$.

[14] A. Boyarsky. Computer orbits. Preprint Concordia Univ., 1984.

[15] Yu. I. Kifer. Small random perturbations of some smooth dynamical systems. Izv. AN SSSR, Ser. Mat. 38(5) (1974), 1091-1115.

[16] G. Keller. Stochastic stability in some chaotic dynamical systems. Monatsh. Math. 94 (1982), 313-333.

[17] W. Parry. Intrinsic Markov chains. Trans. Amer. Math. Soc. 112(1) (1964), 55-66.

[18] M. L. Blank. Small perturbations and stabilization of unstable trajectories. Dept. VINITI, 2201-85, 1985,38 pp.

[19] M. L. Blank. Ergodic properties of discretizations of dynamical systems. Dokl. AN SSSR 278(4) (1984), 779-782.

[20] J. L. Doob. Stochastic Processes IL: Moscow, 1956.

[21] K. Yosida. Functional Analysis Mir: Moscow, 1979.

[22] M. L. Blank. On the conjugacy of a certain class of homeomorphisms to the class of piecewise monotonic maps. Russian Math. Surveys 40(1) (1985), 211-212. 